\title{
Self-consistent molecular dynamics calculation of diffusion in higher n-alkanes
}

\author{
N.D. Kondratyuk ${ }^{1,2}$ G.E. Norman ${ }^{1,2}, \underline{\text { V.V. Stegailov }}{ }^{1,2 *}$ \\ ${ }^{1}$ Joint Institute for High Temperature of RAS, Moscow, Russia \\ ${ }^{2}$ Moscow Institute of Physics and Technology, Dolgoprudny, Russia \\ *stegailov@gmail.com
}

Diffusion is one of the key subjects of molecular modeling and simulation studies. However, there is an unresolved lack of consistency between Einstein-Smoluchowski (E-S) and Green-Kubo (G-K) methods for diffusion coefficient calculations in systems of complex molecules. In this work [1], we analyze this problem for the case of liquid n-triacontane. The non-conventional long-time tails of the velocity autocorrelation function (VACF) are found for this system. Temperature dependence of the VACF tail decay exponent is defined. The proper inclusion of the long-time tail contributions to the diffusion coefficient calculation results in the consistency between G-K and E-S methods. Having considered the major factors influencing the precision of the diffusion rate calculations in comparison with experimental data (system size effects and force field parameters), we point to hydrogen nuclear quantum effects as, presumably, the last obstacle to fully consistent n-alkane description. The first results of the path-integral molecular dynamics will be presented.

The work was supported by the Russian Science Foundation (grant 14-50-00124).

\section{References}

[1] N.D. Kondratyuk, G.E. Norman, V.V. Stegailov: Self-consistent molecular dynamics calculation of diffusion in higher n-alkanes. Journal of Chemical Physics 145204504 (2016) 